PROCEEDINGS OF THE

AMERICAN MATHEMATICAL SOCIETY

Volume 133, Number 11, Pages 3211-3225

S 0002-9939(05)07853-6

Article electronically published on June 20, 2005

\title{
PARAMETRIC BORWEIN-PREISS VARIATIONAL PRINCIPLE AND APPLICATIONS
}

\author{
PANDO GR. GEORGIEV
}

(Communicated by Jonathan M. Borwein)

\begin{abstract}
A parametric version of the Borwein-Preiss smooth variational principle is presented, which states that under suitable assumptions on a given convex function depending on a parameter, the minimum point of a smooth convex perturbation of it depends continuously on the parameter. Some applications are given: existence of a Nash equilibrium and a solution of a variational inequality for a system of partially convex functions, perturbed by arbitrarily small smooth convex perturbations when one of the functions has a non-compact domain; a parametric version of the Kuhn-Tucker theorem which contains a parametric smooth variational principle with constraints; existence of a continuous selection of a subdifferential mapping depending on a parameter.

The tool for proving this parametric smooth variational principle is a useful lemma about continuous $\varepsilon$-minimizers of quasi-convex functions depending on a parameter, which has independent interest since it allows direct proofs of Ky Fan's minimax inequality, minimax equalities for quasi-convex functions, Sion's minimax theorem, etc.
\end{abstract}

\section{INTRODUCTION}

The variational principles in Banach spaces, i.e. Ekeland's variational principle [12], [13] and its smooth generalizations: Borwein-Preiss' [3] and Deville-GodefroyZizler's variational principles [9], [10, [11, are now one of the main tools in nonlinear and non-smooth analysis. Various applications of the Borwein-Preiss smooth variational principle are presented, for instance, in [4], [5], [6], 17].

We present a parametric version of the Borwein-Preiss variational principle, which states that under some conditions we can perturb a convex function depending on a parameter with a smooth convex perturbation in such a way that the minimum point depends continuously on the parameter (Theorem 3.1). Something more, we extend this assertion to the case when the parameterized domains of the convex functions are images of a continuous convex-valued multivalued mapping (Theorem 4.2, (B)).

We show some applications of this result. The first one concerns the existence of a Nash equilibrium for partially convex functions of $n$ variables after arbitrarily small smooth convex perturbations, when the domain of one of the variables is not

Received by the editors May 31, 1999.

2000 Mathematics Subject Classification. Primary 49J35, 49J52; Secondary 46N10.

Key words and phrases. Borwein-Preiss variational principle, Ky Fan's inequality, continuous selections, minimax problems.

(C)2005 American Mathematical Society Reverts to public domain 28 years from publication 
compact (Theorem 4.1 (i)). When $n=2$, this is a "perturbed" version of Sion's minimax theorem [18, showing that the perturbed function has a saddle point. When these functions are equal and Fréchet differentiable, we prove existence of solutions of a related variational inequality defined by arbitrarily small monotone perturbations of their derivatives (Theorem 4.1 (ii)).

The main application is a parametric version of the Kuhn-Tucker theorem (Theorem 4.2. (A)), a particular case of which is a parametric smooth variational principle with constraints (Theorem 4.2, (B)). As a corollary we obtain the existence of a continuous selection of a subdifferential mapping depending on a parameter (Corollary 4.4. (ii), (a)) and, as a particular case, existence of a continuous selection of the mapping $E \ni y \mapsto \partial f(y+\delta B)$ for every $\delta>0$, where $f: E \rightarrow \mathbb{R}$ is a continuous convex function and $B$ is the unit ball in the Banach space $E$ with Fréchet differentiable norm.

The tool for proving this parametric Borwein-Preiss variational principle is a useful lemma (Lemma 2.1) which allows us to find an $\varepsilon$-approximate continuous selection from the $\varepsilon$-level sets of a quasi-convex function depending on a parameter, when the $\varepsilon$-level sets are taken with respect to the images of a lower semi-continuous mapping. This lemma turns out to be a powerful instrument of variational analysis, since it gives simple proofs of: Ky Fan's minimax inequality, extension to quasiconvex functions of minimax equalities (see 2], Theorems 6.3.2 and 6.3.4), Sion's minimax theorem, etc.

\section{Continuous $\varepsilon$-Minimizers}

Let $(E,\|\|$.$) be a Banach space, B$ the open unit ball in $E$ and $Y$ a convex subset of $E$.

Recall that a function $g: Y \rightarrow \mathbb{R}$ is quasi-convex if its sublevel sets $L(g, \alpha):=$ $\{x \in Y: g(x) \leq \alpha\}$ are convex for every $\alpha \in \mathbb{R}$. Equivalently, $g$ is quasi-convex iff $g(\lambda x+(1-\lambda) y) \leq \max \{g(x), g(y)\}$, for every $x, y \in Y, \lambda \in[0,1]$. Recall that a multivalued mapping $F: T \rightarrow E$, where $T$ is a topological space, is lower semicontinuous at $x_{0}$, if for every open $V$ with $V \cap F\left(x_{0}\right) \neq \emptyset$ there exists an open $U \ni x_{0}$ such that $F(x) \cap V \neq \emptyset$ for every $x \in U$. Denote by $\mathbb{R}_{+}$the set of all positive numbers and by $2^{Y}$ the set of all non-empty subsets of the set $Y$.

In the sequel we will use the following lemma, which appears to be a powerful instrument of variational analysis.

Lemma 2.1. Suppose that $X$ is a paracompact topological space, $E$ is a Banach space, $Y \subset E$ is a closed, convex and non-empty subset, $F: X \rightarrow 2^{Y}$ is a lower semi-continuous multivalued mapping with convex non-empty images, $\varepsilon: X \rightarrow \mathbb{R}_{+}$ is a continuous function and the functions $f: X \times Y \rightarrow \mathbb{R}, g: X \rightarrow \mathbb{R}$ satisfy the conditions:

(i) the function $f(x,$.$) is quasi-convex for every x \in X$;

(ii) the function $f(., y)$ is upper semi-continuous for every $y \in Y$;

(iii) $g$ is lower semi-continuous and $g(x) \geq \inf _{y \in(F(x)+\varepsilon(x) B) \cap Y} f(x, y)>-\infty$ for every $x \in X$.

Then

(a) there exists a continuous selection $\varphi_{\varepsilon}: X \rightarrow Y$ of the mapping $F_{\varepsilon}$ (i.e. $\varphi_{\varepsilon}(x) \in F_{\varepsilon}(x)$ for every $\left.x \in X\right)$, where $F_{\varepsilon}(x)=(F(x)+\varepsilon(x) B) \cap Y$, and

$$
f\left(x, \varphi_{\varepsilon}(x)\right)<g(x)+\varepsilon(x) \quad \forall x \in X .
$$


(b) If $F(x)$ is open for every $x \in X$, then there exists a continuous selection $\varphi_{\varepsilon}$ of $F$ satisfying (1).

Proof. (a) Define:

$$
S_{y}=\left\{x \in X: y \in F_{\varepsilon}(x), f(x, y)<g(x)+\varepsilon(x)\right\} .
$$

By lower semi-continuity of $F$ and continuity of $\varepsilon$, by (ii) and (iii) we obtain that this set is open. Obviously $\bigcup_{y \in Y} S_{y}=X$. Since $X$ is paracompact, there exists a locally finite refinement $\left\{U_{j}\right\}_{j \in J}$ of the cover $\left\{S_{y}\right\}_{y \in Y}$. Let $\left\{p_{j}\right\}_{j \in J}$ be a continuous partition of unity, subordinated to this cover. For each $j \in J$ choose $y_{j} \in Y$ such that $\operatorname{supp}\left(p_{j}\right) \subset S_{y_{j}}$. Defining the continuous function $\varphi_{\varepsilon}: X \rightarrow Y$ by

$$
\varphi_{\varepsilon}(x)=\sum_{j \in J} p_{j}(x) y_{j},
$$

it follows that $\varphi_{\varepsilon}$ is a continuous selection of $F_{\varepsilon}$ and satisfies (11).

The assertion (b) is established in a similar way.

Remark 2.2. The existence of an $\varepsilon$-approximate continuous selection of $F$, established by Lemma 2.1, provides half of the proof of Michael's selection theorem (see, for instance, [15], Theorem 4.6).

In the sequel we demonstrate the power of Lemma 2.1 by giving straightforward proofs of some minimax theorems.

Theorem 2.3 (Ky Fan). Suppose that $X$ is a convex, compact and non-empty subset of a topological vector space $E$ and $f: X \times X \rightarrow \mathbb{R}$ is a function with the properties:

(a) $f(., y)$ is lower semi-continuous for every $y \in X$;

(b) $f(x,$.$) is concave for every x \in X$.

Then there exists $x_{0} \in X$ such that

$$
\sup _{y \in X} f\left(x_{0}, y\right) \leq \sup _{y \in X} f(y, y) .
$$

Proof. The essential part of the proof is to establish (2) when $E$ is finite dimensional, since it is easy to extend it using compactness (finite intersection property) to arbitrary topological vector spaces.

Step 1. $X$ is the convex hull of finitely many points, i.e. $X=\operatorname{conv}\left\{y_{i}\right\}_{i=1}^{m}$.

Apply Lemma 2.1 when $F(x)=Y$ to the function $-f$ and the constant function

$$
g(z)=\sup _{x \in X} \inf _{y \in X}(-f)(x, y) \quad \forall z \in X .
$$

So, we obtain a continuous function $\varphi_{n}: X \rightarrow X$ such that

$$
-f\left(x, \varphi_{n}(x)\right) \leq g(x)+1 / n \quad \forall x \in X .
$$

By Brouwer's fixed point theorem there exists a fixed point $x_{n}=\varphi_{n}\left(x_{n}\right)$ of $\varphi_{n}$. Putting $x=x_{n}$ in (3) we obtain

$$
\inf _{x \in X} \sup _{y \in X} f(x, y) \leq f\left(x_{n}, x_{n}\right)+1 / n \leq \sup _{x \in X} f(x, x)+1 / n .
$$

Hence, using (a), we obtain the result.

Step 2. $X$ is an arbitrary non-empty compact convex subset of $E$. 
Define

$$
S(y)=\left\{x \in X: f(x, y) \leq \sup _{z \in X} f(z, z)\right\},
$$

which is a closed subset of $X$. We have proved by Step 1 that for every finite set $Z \subset X$

$$
\bigcap\{S(y): y \in Z\} \neq \emptyset .
$$

By compactness of $X$, we obtain

$$
\bigcap\{S(y): y \in X\} \neq \emptyset,
$$

which completes the proof.

The following theorem is a generalization to the quasi-concave functions of Theorem 6.3.2 in [2] for the non-compact case (in a Banach space) and a modification of Theorem 6.3.4 in 2]. Note that in Theorem 6.3.4 in [2] a compact property is imposed with respect to $X$, but in our case with respect to $Y$.

Theorem 2.4. Let $X$ be a paracompact topological space, $Y$ a convex subset of a Banach space $E$ and $f: X \times Y \rightarrow \mathbb{R}$ a function such that $f(x,$.$) is quasi-concave$ for every $x \in X$ and $f(., y)$ is lower semi-continuous for every $y \in Y$. Then

$$
v^{\sharp}:=\inf _{x \in X} \sup _{y \in Y} f(x, y)=\sup _{C \in \mathcal{C}(X, Y)} \inf _{x \in X} f(x, C(x))=: a,
$$

where $\mathcal{C}(X, Y)$ is the set of all continuous mappings from $X$ to $Y$.

If, in addition, $Y$ is compact, then

$$
v^{\sharp}=\inf _{C \in \mathcal{C}(Y, X)} \sup _{y \in Y} f(C(y), y)=: b .
$$

Proof. By Lemma 2.1 there exists a continuous mapping $\tilde{y}: X \rightarrow Y$ such that

$$
v^{\sharp} \leq f(x, \tilde{y}(x))+\varepsilon \quad \forall x \in X .
$$

Hence $v^{\sharp} \leq a$. The inverse inequality is obvious, so (4) is proven.

Now let $Y$ be compact and let $C \in \mathcal{C}(Y, X)$. By Schauder's fixed point theorem there exists a point $y_{C}=\tilde{y}\left(C\left(y_{C}\right)\right)$. Putting $x=C\left(y_{C}\right)$ in (6) and having in mind that $C \in \mathcal{C}(Y, X)$ is arbitrary, we obtain $v^{\sharp} \leq b$. The inverse inequality is obvious and (5) is proven.

We note that by a similar technique we can give another proof of 2], Theorem 6.3 .8 , for quasi-concave functions.

\section{Parametric Borwein-Preiss variational principle}

Now we present and prove the following parametric Borwein-Preiss variational principle.

Theorem 3.1. Suppose that $X$ is a paracompact topological space, $Y$ is a convex, closed and non-empty subset of a Banach space and the function $f: X \times Y \rightarrow \mathbb{R}$ satisfies the conditions:

(i) for every $x \in X$ the function $f(x,$.$) is convex, lower semi-continuous and:$ if $Y$ is bounded, then $\inf f(x, Y)>-\infty$; if $Y$ is unbounded, then $\inf f(\mathcal{O}, Y)>-\infty$ for some open neighborhood $\mathcal{O}$ of $x$;

(ii) for every $y \in Y$ the function $f(., y)$ is upper semi-continuous; 
(iii) the functions $\left\{f(., y): y \in Y_{0}\right\}$ are equi-lower semi-continuous for every bounded subset $Y_{0} \subset Y$ (this means: for every $x_{0} \in X$ and $\gamma>0$ there exists an open set $\mathcal{O} \ni x_{0}$ such that $f\left(x_{0}, y\right)-f(x, y)<\gamma$ for every $x \in \mathcal{O}$ and every $\left.y \in Y_{0}\right)$.

Let $\varepsilon: X \rightarrow \mathbb{R}_{+}$and $y_{0}: X \rightarrow Y$ be continuous mappings such that

$$
f\left(x, y_{0}(x)\right) \leq \inf f(x, Y)+\varepsilon(x), \quad \forall x \in X .
$$

Then for every $p \geq 1, \alpha>0$ and every continuous function $\lambda: X \rightarrow \mathbb{R}_{+}$, there exist a continuous mapping $v: X \rightarrow Y$, sequences of continuous mappings $y_{n}: X \rightarrow Y$ and sequences of continuous functions $\nu_{n}: X \rightarrow \mathbb{R}_{+}$, such that $y_{n}(x)$ converges uniformly on $x \in X$ to $v(x)$ and

$$
\begin{gathered}
\left\|v(x)-y_{0}(x)\right\|<\lambda(x), \quad \forall x \in X, \\
f(x, v(x))+\Delta(x, v(x)) \leq f(x, y)+\Delta(x, y), \quad \forall x \in X, \forall y \in Y,
\end{gathered}
$$

where

$$
\begin{gathered}
\Delta(x, y)=\sum_{n=0}^{\infty} \mu_{n}(x)\left\|y-y_{n}(x)\right\|^{p} \\
\mu_{n}(x)=\frac{\varepsilon(x)+\alpha}{\lambda(x)^{p}} \nu_{n}(x), \quad \text { for } n \geq 0, \quad \sum_{n=0}^{\infty} \nu_{n}(x)=1 .
\end{gathered}
$$

Proof. Put $\varepsilon_{0}(x)=\varepsilon(x), \mu_{0}(x)=\frac{\varepsilon(x)+\alpha / 2}{\lambda(x)^{p}}, \nu_{0}(x)=\frac{\mu_{0}(x) \lambda(x)^{p}}{\varepsilon(x)+\alpha}$ and for $n \geq 1$, put $\nu_{n}(x)=\frac{1-\nu_{0}(x)}{2^{n}}, \mu_{n}(x)=\frac{\varepsilon(x)+\alpha}{\lambda(x)^{p}} \nu_{n}(x), \lambda_{n}(x)=\left(\frac{\varepsilon_{n}(x)+\varepsilon_{n+1}(x)}{\mu_{n}(x)}\right)^{\frac{1}{p}}, \varepsilon_{n}(x)=$ $\beta(x) 2^{-2 n}$, where $\beta: X \rightarrow \mathbb{R}_{+}$is a continuous function such that

$$
\left(\frac{5 \beta(x)}{2 \alpha}\right)^{1 / p} \cdot \frac{1}{2^{1 / p}-1}+\left(\frac{\varepsilon(x)+\beta(x) / 4}{\varepsilon(x)+\alpha / 2}\right)^{1 / p}<1 .
$$

Then $\nu_{0} \in(0,1), \sum_{n=0}^{\infty} \nu_{n}(x)=1, \lambda_{0}(x)=\left(\frac{\varepsilon(x)+\beta(x) / 4}{\varepsilon(x)+\alpha / 2}\right)^{1 / p} \lambda(x), \lambda_{n}(x)=$ $\left(\frac{5 \beta(x)}{\alpha}\right)^{1 / p} 2^{(-n-1) / p} \lambda(x)$ and

$$
\sum_{n=0}^{\infty} \lambda_{n}(x)<\lambda(x), \quad \forall x \in X .
$$

Applying Lemma 2.1 for $g(x)=\inf f_{n}(x, Y), n=1,2, \ldots, F(x)=Y$, we define inductively sequences of functions $f_{n}: X \times Y \rightarrow \mathbb{R}$ and continuous mappings $y_{n}: X \rightarrow Y$, satisfying for every $n \geq 0$,

$$
\begin{gathered}
f_{n+1}(x, y)=f_{n}(x, y)+\mu_{n}(x)\left\|y-y_{n}(x)\right\|^{p}, \quad f_{0}=f, \\
f_{n}\left(x, y_{n}(x)\right) \leq \inf f_{n}(x, Y)+\varepsilon_{n}(x), \quad \forall x \in X .
\end{gathered}
$$

We need to check the condition (iii) of Lemma 2.1 in order to justify its applicability.

We will prove that $g$ is lower semi-continuous. Consider the case when $Y$ is unbounded (the case when $Y$ is bounded is similar). Let $x_{0} \in X$ and $\gamma>0$ be given. Take any $z_{0} \in Y$. By upper semi-continuity of $f_{n}\left(., z_{0}\right)$ and continuity of $y_{n}$ at $x_{0}$, there exists an open neighborhood $\mathcal{O}$ of $x_{0}$ such that $f_{n}\left(x, z_{0}\right)<f_{n}\left(x_{0}, z_{0}\right)+\gamma$ for every $x \in \mathcal{O}$ and $y_{n}(\mathcal{O})$ is bounded. Define

$$
Y_{n}=\bigcup_{x \in \mathcal{O}} L_{n}(x), \text { where } L_{n}(x)=\left\{y \in Y: f_{n}(x, y)<\inf _{z \in Y} f_{n}(x, z)+\gamma\right\} .
$$


We will prove that $Y_{n}$ is bounded. Assume the contrary: there are sequences $\left\{x_{m}\right\}_{m=1}^{\infty} \subset \mathcal{O}$ and $\left\{z_{m}\right\}_{m=1}^{\infty}, z_{m} \in L_{n}\left(x_{m}\right)$ such that $\left\|z_{m}\right\| \rightarrow \infty$. We have

$$
\begin{aligned}
-\infty & <f_{n-1}\left(x_{m}, z_{m}\right)+\mu_{n}\left\|z_{m}-y_{n}\left(x_{m}\right)\right\|^{p} \quad \text { (by (i)) } \\
& <\inf _{z \in Y} f_{n}\left(x_{m}, z\right)+\gamma \\
& <f_{n}\left(x_{m}, z_{0}\right)+\gamma \\
& <f_{n}\left(x_{0}, z_{0}\right)+2 \gamma .
\end{aligned}
$$

This leads to a contradiction when $m \rightarrow \infty$. The boundedness of $Y_{n}$ guarantees that the functions $\left\{\|y-.\|^{p}: y \in Y_{n}\right\}$ are equi-Lipschitz, and by (iii), there exists an open neighborhood $\mathcal{O}_{1} \subset \mathcal{O}$ of $x_{0}$ such that

$$
f_{n}\left(x_{0}, y\right)-f_{n}(x, y)<\gamma \quad \forall y \in Y_{n}, \forall x \in \mathcal{O}_{1} .
$$

For any $x \in \mathcal{O}_{1}$ take $y_{x} \in L_{n}(x)$. Then we have

$$
\begin{aligned}
g\left(x_{0}\right)-g(x) & =\inf _{y \in Y} f_{n}\left(x_{0}, y\right)-\inf _{y \in Y} f_{n}(x, y) \\
& \leq f_{n}\left(x_{0}, y_{x}\right)-f_{n}\left(x, y_{x}\right)+\gamma \\
& \leq 2 \gamma,
\end{aligned}
$$

which proves the lower semi-continuity of $g$ and the correctness of (13), (14).

Now by (13) and (14) we obtain

$$
\begin{aligned}
\mu_{n}(x)\left\|y_{n+1}(x)-y_{n}(x)\right\|^{p}= & f_{n+1}\left(x, y_{n+1}(x)\right)-f_{n}\left(x, y_{n+1}(x)\right) \\
= & f_{n+1}\left(x, y_{n+1}(x)\right)-f_{n+1}\left(x, y_{n}(x)\right) \\
& +f_{n}\left(x, y_{n}(x)\right)-f_{n}\left(x, y_{n+1}(x)\right) \\
< & \varepsilon_{n+1}(x)+\varepsilon_{n}(x) .
\end{aligned}
$$

Hence, by definition of $\lambda_{n}(x),\left\|y_{n+1}(x)-y_{n}(x)\right\|<\lambda_{n}(x)$ and, therefore,

$$
\left\|y_{n+m}(x)-y_{n}(x)\right\|<\sum_{i=0}^{m-1} \lambda_{n+i}(x) .
$$

By (12) and continuity of the functions $\lambda_{i}, i=0,1, \ldots$, it follows that $\left\{y_{n}(x)\right\}_{n \geq 0}$ is a Cauchy sequence, converging uniformly on a neighborhood $\mathcal{O}_{x}$ of $x$ to $v(x)$ for every $x \in X$, which implies that the mapping $v: X \rightarrow Y$ is continuous. By (15) for $m \rightarrow \infty$ and $n=0$ and by (12) it follows that $\left\|v(x)-y_{0}(x)\right\|<\sum_{i=0}^{\infty} \lambda_{i}(x)<\lambda(x)$, which is (8).

We will prove that for every $x \in X, v(x)$ is a minimum point for the function

$$
\tilde{f}(x, .)=f(x, .)+\sum_{n=0}^{\infty} \mu_{n}(x)\left\|.-y_{n}(x)\right\|^{p} .
$$

Let $\gamma>0, x \in X$ be given. Since $\tilde{f}(x,$.$) is lower semi-continuous, there exists$ $\delta>0$ such that

$$
\tilde{f}(x, v(x))<\tilde{f}(x, y)+\frac{\gamma}{3}, \quad \forall y \in B(v(x), \delta) .
$$

Choose $n$ sufficiently large such that $\varepsilon_{n}(x)<\gamma / 3,\left\|y_{n}(x)-v(x)\right\|<\delta$ and

$$
\sum_{k=n+1}^{\infty} \mu_{k}(x)\left\|y_{n}(x)-y_{k}(x)\right\|^{p}<\frac{\gamma}{3}
$$


for every $x \in X$. For every $y \in Y$, using (16), (13) and (14), we can write:

$$
\begin{aligned}
\tilde{f}(x, v(x)) & <\tilde{f}\left(x, y_{n}(x)\right)+\frac{\gamma}{3} \\
& =f_{n}\left(x, y_{n}(x)\right)+\sum_{k=n+1}^{\infty} \mu_{k}(x)\left\|y_{n}(x)-y_{k}(x)\right\|^{p}+\frac{\gamma}{3} \\
& \leq f_{n}(x, y)+\varepsilon_{n}(x)+\frac{\gamma}{3}+\frac{\gamma}{3} \\
& <\tilde{f}(x, y)+\gamma .
\end{aligned}
$$

This proves (9) and the theorem.

Remark 3.2. It is clear that if we replace in the above theorem $\Delta(x, y)$ with $\Delta(x, y)+$ $\varepsilon\|y-v(x)\|^{p}$ with any $\varepsilon>0$, we will obtain that $v(x)$ is a strong minimum of the perturbed function, i.e. every minimizing sequence converges to $v(x)$.

Remark 3.3. If we choose $p=1$ in the above theorem and take into account Remark 3.2, we will obtain a parametric version of Ekeland's variational principle in a strong form, i.e. with convergence of every minimizing sequence.

\section{Applications of the parametric Borwein-Preiss VARIATIONAL PRINCIPLE}

In the next theorem we establish the existence of a Nash equilibrium for convex functions after arbitrarily small smooth convex perturbations, when the domain of one of the variables is not compact, and existence of solutions for a related variational inequality.

Theorem 4.1. Let $X_{i}, i=2, \ldots, n$, be convex, compact and non-empty subsets of the Banach spaces $E_{i}$, and let $X_{1}$ be a convex, closed and non-empty subset of a Banach space $E_{1}$. Denote $X=X_{1} \times \cdots \times X_{n}, E=E_{1} \times \cdots \times E_{n}, x=\left(x_{1}, \ldots, x_{n}\right)$, $x_{\hat{i}}=\left(x_{1}, \ldots, x_{i-1}, x_{i+1}, \ldots, x_{n}\right), X_{\hat{i}}=X_{1} \times \cdots \times X_{i-1} \times X_{i+1} \times \cdots \times X_{n}, \forall i=$ $1, \ldots, n$. Assume that the functions $f_{i}: E \rightarrow \mathbb{R}, i=1, \ldots, n$, are convex with respect to $x_{i} \in X_{i}$, bounded below on $X$, the functions $\left\{f_{i}\left(\ldots, x_{i}, \ldots\right): x_{i} \in X_{i}\right\}$ are upper semi-continuous and equi-lower semi-continuous on $X_{\hat{i}}$ for every $i=2, \ldots, n$ and the functions $\left\{f_{1}\left(x_{1}, \ldots\right): x_{1} \in Y\right\}$ are upper semi-continuous and equi-lower semi-continuous on $X_{\hat{1}}$ for every bounded subset $Y \subset X_{1}$. Then for every $\varepsilon>0$ there exist points $\bar{x}_{i} \in X_{i}$ and convex functions $b_{i}: X_{i} \rightarrow \mathbb{R}, i=1, \ldots, n$, which are Lipschitz with a Lipschitz constant less than $\varepsilon$ (except for $b_{1}$, which is Lipschitz on the bounded sets, with a Lipschitz constant less than $\varepsilon$ on a neighrborhood of $\left.\bar{x}_{1}\right)$, which are differentiable, if the norm of $E_{i}$ is differentiable on $E_{i} \backslash\{0\}$, such that:

(i) the point $\bar{x}=\left(\bar{x}_{1}, \ldots, \bar{x}_{n}\right)$ is a Nash equilibrium for the functions $f_{i}(x)+$ $b_{i}\left(x_{i}\right), i=1, \ldots, n ;$ i.e.

$$
\begin{aligned}
& f_{i}\left(\bar{x}_{1}, \ldots, \bar{x}_{i}, \ldots, \bar{x}_{n}\right)+b_{i}\left(\bar{x}_{i}\right) \\
& \quad \leq f_{i}\left(\bar{x}_{1}, \ldots, x_{i}, \ldots, \bar{x}_{n}\right)+b_{i}\left(x_{i}\right), \quad \forall x_{i} \in X_{i}, \forall i=1, \ldots, n,
\end{aligned}
$$

(ii) if all the functions $f_{i}$ are equal, $f_{i}=f, i=1, \ldots, n, f$ is Fréchet differentiable on $X$, and if the norms of $E_{i}$ are Fréchet differentiable on $E_{i} \backslash\{0\}$, then the $b_{i}$ are Fréchet differentiable and

$$
\left\langle f^{\prime}(\bar{x})+b^{\prime}(\bar{x}), x-\bar{x}\right\rangle \geq 0 \quad \forall x \in X
$$

where $b^{\prime}(\bar{x})=\left(b_{1}^{\prime}\left(\bar{x}_{1}\right), \ldots, b_{n}^{\prime}\left(\bar{x}_{n}\right)\right)$. 
Proof. (i) By Theorem 3.1 for every $i=1, \ldots, n$ there exists a continuous mapping $y_{i}: X_{\hat{i}} \rightarrow X_{i}$ and a continuous function $\Delta_{i}: X \rightarrow \mathbb{R}$ of type (10), which is differentiable, convex and Lipschitz with respect to the variable $x_{i}$ on $X_{i}$ with a Lipschitz constant less than $\varepsilon$ (except for $\Delta_{1}$, which is Lipschitz with respect to $x_{1}$ on the bounded subsets of $X_{1}$ and has a Lipschitz constant less than $\varepsilon$ on a neighborhood of $\bar{x}_{1}$ ), such that

$$
\begin{aligned}
& \left(f_{i}+\Delta_{i}\right)\left(x_{1}, \ldots, y_{i}\left(x_{\hat{i}}\right), \ldots, x_{n}\right) \\
& \quad \leq\left(f_{i}+\Delta_{i}\right)\left(x_{1}, \ldots, x_{n}\right), \quad \forall x_{i} \in X_{i}, \forall i=1, \ldots, n .
\end{aligned}
$$

The composition mapping

$$
X_{2} \times \cdots \times X_{n} \ni x_{\hat{1}} \mapsto\left(y_{2}\left(\varphi_{2}\left(x_{\hat{1}}\right)\right), \ldots, y_{n}\left(\varphi_{n}\left(x_{\hat{1}}\right)\right)\right) \in X_{2} \times \cdots \times X_{n},
$$

where $\varphi_{i}\left(x_{\hat{1}}\right)=\left(y_{1}\left(x_{\hat{1}}\right), x_{2}, \ldots, x_{i-1}, x_{i+1}, \ldots, x_{n}\right), i=2, \ldots, n$, is a continuous mapping from the compact convex set $X_{2} \times \cdots \times X_{n}$ to itself and by Schauder's fixed point theorem it has a fixed point $\bar{x}_{\hat{1}}=\left(\bar{x}_{2}, \ldots, \bar{x}_{n}\right)$. If we put $\bar{x}_{1}=y_{1}\left(\bar{x}_{\hat{1}}\right)$ and $b_{i}\left(x_{i}\right):=\Delta\left(\bar{x}_{1}, \ldots, x_{i}, \ldots, \bar{x}_{n}\right)$, then $\bar{x}_{i}=y_{i}\left(\bar{x}_{\hat{i}}\right)$ for every $i=2, \ldots, n$, and from (19) we obtain (17).

(ii) By (17) we obtain

$$
\left\langle f_{x_{i}}^{\prime}(\bar{x})+b^{\prime}\left(\bar{x}_{i}\right), x_{i}-\bar{x}_{i}\right\rangle \geq 0 \quad \forall x_{i} \in X_{i},
$$

and after summing in (20) for $i=1, \ldots, n$, we obtain (18).

We note that by the same technique for $n=2$, applying Lemma 2.1 instead of Theorem 3.1 we can prove Sion's minimax theorem [18] (this is an interesting exercise, left to the reader).

In the next theorem we establish continuous dependence of Lagrange multipliers of a constraint minimization problem depending on a parameter.

Suppose that a hypothesis $(\mathrm{H})$ consisting of the following conditions is satisfied:

$\left(H_{a}\right) P$ is a paracompact topological space,

$\left(H_{b}\right) E$ is a Banach space, $C \subset E$ is a closed, convex and non-empty set, $\varepsilon_{0}>0$ is given, $C_{0}=C+\varepsilon_{0} B$,

$\left(H_{c}\right)$ the functions $f, g_{i}: P \times C_{0} \rightarrow \mathbb{R}, i=1, \ldots, n$, are convex and continuous with respect to the second variable,

$\left(H_{d}\right)$ the families of functions $\left\{f(., x): x \in C_{0}\right\},\left\{g_{i}(., x): x \in C_{0}\right\}, i=1, \ldots, n$, are equi-continuous,

$\left(H_{e}\right)$ the multivalued mapping $\mathcal{C}: P \rightarrow 2^{C}$ has closed convex and non-empty values and is lower semi-continuous and Hausdorff upper semi-continuous.

$\left(H_{f}\right)$ the set $X(p)=\left\{x \in \mathcal{C}(p): g_{i}(p, x) \leq 0, i=1, \ldots, n\right\}$ is non-empty for every $p \in P$.

Consider the following parameterized minimization problem $\mathcal{P}$ :

minimize $f(p, x)$ with respect to $x$ for every $p \in P$,

under constraints: $x \in X(p)$.

Denote $g(p, x)=\left(g_{1}(p, x), \ldots, g_{n}(p, x)\right)$ and $\operatorname{dist}_{Y}(x)=\inf _{y \in Y}\|x-y\|$, the distance from a point $x$ to a set $Y$.

Theorem 4.2 (Parametric Kuhn-Tucker theorem). Assume that the hypothesis $(\mathrm{H})$ is satisfied and the norm of the Banach space $E$ is Fréchet differentiable on $E \backslash\{0\}$. Assume that the continuous functions $\varepsilon, \lambda: X \rightarrow \mathbb{R}_{+}, \gamma: X \rightarrow \mathbb{R}$ and the 
numbers $\alpha>0, q \geq 1$ are given,

$$
0 \leq \gamma(p)<\frac{\lambda(p)}{2 q 5^{q-1}}
$$

the functions $f(p,),. g_{i}(p,),. i=1, \ldots, n$, are bounded on $u(p)+3 \lambda(p) B$ for every $p \in P, u: P \rightarrow C$ is a continuous mapping satisfying

$$
u(p) \in X_{\gamma}(p) \quad \text { and } \quad f(p, u(p))<\inf _{x \in X_{\gamma}(p)} f(p, x)+\varepsilon(p) \quad \forall p \in P,
$$

where $X_{\gamma}(p)=\left\{x \in \mathcal{C}(p)+\gamma(p) B: g_{i}(p, x) \leq 0, i=1, \ldots, n\right\}$. Then there exist a continuous selection $v$ of $\mathcal{C}$ (solution mapping), continuous mappings $\mu: P \rightarrow \mathbb{R}_{+}$, $r: P \rightarrow \mathbb{R}_{+}^{n}$ (Lagrange multipliers mappings) and a continuous function $K: P \rightarrow$ $\mathbb{R}_{+}$such that:

(A) for every $p \in P$ the following conditions are satisfied:

(i) $\|(\mu(p), r(p))\|=1, \mu(p) \geq 0, r_{i}(p) \geq 0, i=1, \ldots, n$,

(ii) $r_{i}(p) g_{i}(p, u(p))=0 \quad$ for every $i=1, \ldots, n$,

(iii) $\|v(p)-u(p)\|<\lambda(p)$,

(iv) the function $\mu(p) f(p, x)+\langle r(p), g(p, x)\rangle+\Delta(p, x)$ attains its minimum over $\mathcal{C}(p)$ at $v(p)$,

$(\mathrm{v})-\Delta_{x}^{\prime}(p, v(p)) \in \partial_{x} L(p, v(p), \mu(p), r(p), K(p))$,

where $\partial_{x} L(p, v, \mu, r, K)$ is the subdifferential of $L$ with respect to the second variable, $L$ is the Lagrange function given by

$$
L(p, x, \mu, r, K)=\mu f(p, x)+\langle r, g(p, x)\rangle+K \operatorname{dist}_{\mathcal{C}(p)}(x),
$$

$\Delta(p,$.$) is a convex, Fréchet differentiable function, \Delta(., x)$ is continuous:

$$
\begin{gathered}
\Delta(p, x)=\sum_{i=0}^{\infty} \mu_{i}(p)\left\|x_{i}(p)-x\right\|^{q}, \\
\mu_{i}(p)=\frac{\varepsilon(p)+\alpha+K(p) \gamma(p)}{\lambda(p)^{q}} \nu_{i}(p), \quad \text { for } \quad i \geq 0, \quad \sum_{i=0}^{\infty} \nu_{i}(p)=1,
\end{gathered}
$$

$\nu_{i}, x_{i}: P \rightarrow C_{0}$ are continuous mappings, and $x_{i}(p)$ converges uniformly on $p \in P$ to $v(p)$.

(B) If $\mu(p) \neq 0$ for $p \in P_{0} \subset P$, then $v(p)$ is a solution of the following minimization problem for every $p \in P_{0}$ :

$$
\begin{aligned}
& \text { minimize } f(p, x)+\sum_{i=0}^{\infty} \mu_{i}(p)\left\|x_{i}(p)-x\right\|^{q} \quad \text { with respect to } x \\
& \text { under constraints } x \in X(p) .
\end{aligned}
$$

Remark 4.3. The assertion (B) in the above theorem presents a parametric BorweinPreiss variational principle with constraints. A sufficient condition for $\mu(p) \neq 0, p \in$ $P_{0}$ is the following Slater condition (see, for instance, [8], Proposition 6.3.1):

$$
\forall p \in P_{0}, \exists z(p) \in \mathcal{C}(p): g_{i}(p, z(p))<0, \quad \forall i=1, \ldots, n .
$$

The assumption for existence of a continuous mapping $u: P \rightarrow C$ satisfying (22) is fulfilled under some conditions (see Lemma4.6). Also, the existence of a continuous $\lambda$ satisfying the conditions of Theorem 4.2 is guaranteed if $P$ is a metric space (see the proof of Corollary (4.4), (ii)). 
Proof of Theorem 4.2. (A) Put

$$
L_{0}(p)=\sup |f(p, u(p)+3 \lambda(p) B)|
$$

and

$$
L_{i}(p)=\sup \left|g_{i}(p, u(p)+3 \lambda(p) B)\right| .
$$

By a basic lemma of convex analysis (see for instance [15], Lemma 5.23, page 742), the functions $f(p,$.$) and g_{i}(p,),. i=1, \ldots, n$, are Lipschitz on $u(p)+2 \lambda(p) B$ with Lipschitz constants $L_{f}(p)=2 L_{0}(p) / \lambda(p)$ and $L_{g_{i}}(p)=2 L_{i}(p) / \lambda(p), i=1, \ldots, n$, respectively. Put

$$
\tilde{L}(p)=L_{f}(p)+\sum_{i=1}^{n} L_{g_{i}}(p), \quad Q(p)=q 5^{q-1} / \lambda(p),
$$

and

$$
L(p)=\frac{\tilde{L}(p)+(\varepsilon(p)+\alpha) Q}{1-2 \gamma(p) Q(p)}, \quad K(p)=2 L(p)
$$

By equi-continuity assumptions on $\left\{f(., x): x \in C_{0}\right\}$ and $\left\{g_{i}(., x): x \in C_{0}\right\}, i=$ $1, \ldots, n$, and continuity with respect to the second argument, it is straightforward to prove that the functions $L_{i}, i=0, \ldots, n$, are continuous; therefore $K$ is continuous too.

Further we use an idea from the proof of [8], Theorem 6.1.1. Put

$$
T=\left\{t=(\mu, r) \in \mathbb{R}^{1+n}: \mu \geq 0, r \geq 0,\|(\mu, r)\|=1\right\}
$$

and

$$
F(p, y)=\max _{t \in T}\{\mu(f(p, y)-f(p, u(p))+\varepsilon(p))+\langle r, g(p, y)\rangle\}+K(p) \operatorname{dist}_{\mathcal{C}(p)}(y) .
$$

Because of $g_{i}(p, u(p)) \leq 0$, we have $F(p, u(p)) \leq \varepsilon(p)+K(p) \operatorname{dist}_{\mathcal{C}(p)}(u(p))<\varepsilon(p)+$ $K(p) \gamma(p)$. Also, $F(p, x)>0$ for $x \in C_{0}$. Indeed, if $F(p, y) \leq 0$ for some $y \in C_{0}$, then $g_{i}(p, y) \leq 0$ for every $i=1, \ldots, n, y \in \mathcal{C}(p)$ and $f(p, y) \leq f(p, u(p))-\varepsilon(p)<$ $\inf f(p, X(p))$, a contradiction. So we have

$$
F(p, u(p)) \leq \inf F\left(p, C_{0}\right)+\varepsilon(p)+K(p) \gamma(p) \quad \forall p \in P .
$$

We will show that $F$ satisfies the condition of Theorem 3.1 Firstly we will establish that the functions $\left\{F(., y): y \in Y_{0}\right\}$ are equi-lower semi-continuous for any bounded subset $Y_{0}$ of $C_{0}$.

It is straightforward to check that the functions $\left\{\operatorname{dist}_{\mathcal{C}(.)}(y): y \in C_{0}\right\}$ are equi-continuous. Let $\delta>0$ be given. By (d), by equi-continuity of the family $\left\{\operatorname{dist}_{\mathcal{C}(.)}(y): y \in C_{0}\right\}$, continuity of $u, K$ and $L_{f}\left(., u\left(p_{0}\right)\right)$, there exists an open set $\mathcal{O} \ni p_{0}$ such that for any $p \in \mathcal{O}$ and any $y \in Y_{0}$ (using the boundedness of $Y_{0}$ ) we 
obtain:

$$
\begin{aligned}
F\left(p_{0}, y\right)-F(p, y) \leq & \max _{t \in T}\left\{\mu\left(f\left(p_{0}, y\right)-f\left(p_{0}, u\left(p_{0}\right)\right)-f(p, y)+f(p, u(p))\right)\right. \\
& \left.+\left\langle r, g\left(p_{0}, y\right)-g(p, y)\right\rangle\right\}+\left(K\left(p_{0}\right)-K(p)\right) \operatorname{dist}_{\mathcal{C}\left(p_{0}\right)}(y) \\
& +K(p)\left(\operatorname{dist}_{\mathcal{C}\left(p_{0}\right)}(y)-\operatorname{dist}_{\mathcal{C}(p)}(y)\right) \\
\leq & +f(p, u(p))-f\left(p, u\left(p_{0}\right)\right)+f\left(p, u\left(p_{0}\right)\right)-f\left(p_{0}, u\left(p_{0}\right)\right) \\
& +n \delta+2 \delta \\
\leq & +L_{f}\left(p, u\left(p_{0}\right)\right)\left\|u(p)-u\left(p_{0}\right)\right\|+\delta \\
& +n \delta+2 \delta \\
\leq & (n+5) \delta .
\end{aligned}
$$

This shows that the functions $\left\{F(., x): x \in Y_{0}\right\}$ are equi-lower semi-continuous. Analogically we prove that the functions $\left\{F(., x), x \in Y_{0}\right\}$ are equi-upper semicontinuous. Obviously $F(p,$.$) is convex.$

Now we apply the parametric Borwein-Preiss variational principle (Theorem 3.1) and obtain: there exist continuous mappings $v, x_{i}: P \rightarrow C_{0}$ and continuous functions $\nu_{i}: X \rightarrow \mathbb{R}_{+}$such that $x_{i}(p)$ converges uniformly on $p \in P$ to $v(p)$ $\left(i=0,1, \ldots, x_{0}=u\right)$, and

$$
\begin{gathered}
\|v(p)-u(p)\|<\lambda(p), \quad \forall p \in P, \\
F(p, v(p))+\Delta(p, v(p)) \leq F(p, y)+\Delta(p, y), \quad \forall p \in P, \forall y \in C_{0},
\end{gathered}
$$

where

$$
\begin{gathered}
\Delta(p, y)=\sum_{i=0}^{\infty} \mu_{i}(p)\left\|y-x_{i}(p)\right\|^{q} \\
\mu_{i}(p)=\frac{\varepsilon(p)+K(p) \gamma(p)+\alpha}{\lambda(p)^{q}} \nu_{i}(p), \quad \text { for } \quad i \geq 0, \quad \sum_{i=0}^{\infty} \nu_{i}(p)=1 .
\end{gathered}
$$

Equation (29) is (iii).

We will prove that $v(p) \in \mathcal{C}(p)$ for every $p \in P$.

Assume the contrary: $v(p) \notin \mathcal{C}(p)$ for some $p \in P$. Because of (29), $\lambda(p)+\gamma(p)>$ $\|v(p)-u(p)\|+\operatorname{dist}_{\mathcal{C}(y)}(u(p)) \geq \operatorname{dist}_{\mathcal{C}(p)}(v(p))$, so $\lambda(p)+\gamma(p)-\operatorname{dist}_{\mathcal{C}(p)}(v(p))>0$. Take $\varepsilon(p) \in\left(0, \lambda(p)+\gamma(p)-\operatorname{dist}_{\mathcal{C}(p)}(v(p))\right.$ and $y(p) \in \mathcal{C}(p)$ such that $\varepsilon(p)<$ $\operatorname{dist}_{\mathcal{C}(p)}(v(p))$ and $\|v(p)-y(p)\|<\operatorname{dist}_{\mathcal{C}(p)}(v(p))+\varepsilon(p)$. Then $\|v(p)-y(p)\|<$ $\lambda(p)+\gamma(p)$ and by (29) $),\|u(p)-y(p)\|<2 \lambda(p)+\gamma(p)$. Put

$$
\varphi(p, y)=F(p, y)-K(p) \operatorname{dist}_{\mathcal{C}(p)}(y)+\Delta(p, y) .
$$

Note that $L(p)$ (given in (27)) is a Lipschitz constant of $\varphi(p,$.$) in \mathcal{O}(p):=u(p)+$ $(2 \lambda(p)+\gamma(p)) B$. Indeed, the norm of the derivative $\Delta^{\prime}(p, y)$ is bounded by $(\varepsilon(p)+\alpha+K(p) \gamma(p)) Q(p)$ on $\mathcal{O}(p)$, so $(\varepsilon(p)+a+K(p) \gamma(p)) Q(p)$ is a Lipschitz constant of $\Delta(p,$.$) on \mathcal{O}(p)$. A Lipschitz constant of $F(p,)-.K(p) \operatorname{dist}_{\mathcal{C}(p)}(y)$ on $\mathcal{O}(p)$ is $\tilde{L}(p)$, so $\tilde{L}(p)+(\varepsilon(p)+\alpha+K(p) \gamma(p)) Q(p)=L(p)$ is a Lipschitz constant of $\varphi(p,$.$) on \mathcal{O}(p)$. Therefore, $L(p)$ is a Lipschitz constant of $\varphi(p,$.$) in a neighborhood,$ say $U$, of $y(p)$. Put $x_{\nu}(p)=\nu v(p)+(1-\nu) y(p)$ and take sufficiently small $\nu>0$ 
such that $x_{\nu}(p) \in U$. From (30) for $x=y(p)$ we obtain:

$$
\begin{aligned}
\varphi\left(p, x_{\nu}(p)\right) & \leq \nu \varphi(p, v(p))+(1-\nu) \varphi(p, y(p)) \\
& \leq \varphi(p, y(p))-\nu K(p) \operatorname{dist}_{\mathcal{C}(p)}(v(p)) .
\end{aligned}
$$

Hence

$$
\begin{aligned}
\nu K(p) \operatorname{dist}_{\mathcal{C}(p)}(v(p)) & \leq \varphi(p, y(p))-\varphi\left(p, x_{\nu}(p)\right) \\
& \leq L(p)\left\|y(p)-x_{\nu}(p)\right\| \\
& =\nu L(p)\|v(p)-y(p)\| \\
& \leq \nu L(p)\left(\operatorname{dist}_{\mathcal{C}(p)}(v(p))+\varepsilon(p)\right),
\end{aligned}
$$

and remembering that $K(p)=2 L(p)$, we obtain $\operatorname{dist}_{\mathcal{C}(p)}(v(p))<\varepsilon(p)$, a contradiction.

So we proved that $v(p) \in \mathcal{C}(p)$ for every $p \in P$. This means in particular that $v(p)$ is an interior point of $C_{0}$ (remember that $\left.C_{0}=C+\varepsilon_{0} B\right)$. Since $F(p, v(p))>0$ and the Euclidean norm is strictly convex, there exists a unique vector $t(p)=(\mu(p), r(p))$ at which the maximum in (28) is attained for $y=v(p)$. Since $\|t(p)\|=1$, (i) is proved. Note that $r_{i}(p)=0$, if $g_{i}(p, v(p))<0$, which gives (ii). Obviously the mapping $p \mapsto t(p)$ is continuous. Now from (30) we obtain (iv) and by Fermat's rule, using the differentiability of the norm, we obtain (v).

(B) The assertion follows from (i), (ii), (iv) and the non-parametric Kuhn-Tucker theorem (see for instance [1, section 1.3.3).

We will present corollaries when the inequality constraints in the minimization problem $\mathcal{P}$ are missing.

Corollary 4.4. (i) Assume that the assumptions of Theorem 4.2 are satisfied, as the functions $g_{i}$ are missing. Then the mapping $p \mapsto \partial f(p, v(p))+N_{\mathcal{C}(p)}(v(p))$ has a continuous selection, where $N_{Y}(y)$ means the normal cone to the convex set $Y$ at the point $y \in Y$ and $\partial f(p, x)$ is the subdifferential of $f(p,$.$) at x$.

(ii) Let $M$ be a metric space, let $E$ be a Banach space with Fréchet differentiable norm on $E \backslash\{0\}$, let $\varphi: M \times E \rightarrow \mathbb{R}$ be a convex continuous function with respect to the second variable and let the functions $\{f(., x): x \in Y\}$ be equi-continuous for every bounded $Y \subset E$. Then for every $\delta>0$ and every continuous mapping $u: M \rightarrow E$ there exist continuous mappings $v: M \rightarrow E$ and $s: M \rightarrow E^{*}$ such that

(a) $s(p) \in \partial f(p, v(p)), \quad \forall p \in M$,

(b) $\|u(p)-v(p)\|<\delta, \quad \forall p \in M$.

In particular,

(c) for every convex continuous function $f: E \rightarrow \mathbb{R}$ and for every $\delta>0$, there exists a continuous selection of the mapping $E \ni p \mapsto \partial f(p+\delta B)$.

Proof. (i) The assertion follows from (v) of Theorem 4.2, having in mind that $\partial \operatorname{dist}_{\mathcal{C}(p)}(x)=\bar{B} \cap N_{\mathcal{C}(p)}(x)$ (see, for instance, [7, page 57, Exercise 12 (d) (iii)).

(ii) For any $p \in M$ define $\alpha_{0}(p)=\sup \{\alpha>0: f(p, x)<f(p, u(p))+4, \quad \forall x \in$ $B(u(p), \alpha)\}, \alpha(p)=\alpha_{0}(p)$ if $\alpha_{0}(p)<+\infty$ and $\alpha(p)=1$ otherwise. By continuity of $f(p,$.$) we have \alpha(p)>0$. Take any $p_{0} \in M$. By continuity of $f(p,),. u($.$) and$ equi-continuity of $\{f(., x): x \in Y\}$ for any bounded $Y$, there exists $\delta\left(p_{0}\right)>0$ and $\alpha_{1}\left(p_{0}\right) \in(0,1)$ such that $f(p, x)<f\left(p_{0}, x\right)+1<f\left(p_{0}, u\left(p_{0}\right)\right)+2<f\left(p_{0}, u(p)\right)+$ $3<f(p, u(p))+4$ and $\left\|u(p)-u\left(p_{0}\right)\right\|<\alpha_{1}\left(p_{0}\right) / 2$ for every $p \in B\left(p_{0}, \delta\left(p_{0}\right)\right)$ and 
$x \in B\left(u\left(p_{0}\right), \alpha_{1}\left(p_{0}\right)\right)$. Then the inclusion $B\left(u(p), \alpha_{1}\left(p_{0}\right) / 2\right) \subset B\left(u\left(p_{0}\right), \alpha_{1}\left(p_{0}\right)\right)$ for $p \in B\left(p_{0}, \delta\left(p_{0}\right)\right)$ implies $\alpha(p) \geq \alpha_{1}\left(p_{0}\right) / 2$. Therefore the lower semi-continuous envelope of $\alpha, \beta\left(p_{0}\right)=\sup _{\varepsilon>0} \inf _{p \in B\left(p_{0}, \varepsilon\right)} \alpha(p)$ is positive, i.e. $\beta\left(p_{0}\right)>0$ for every $p_{0} \in M$. Applying a theorem of Dowker (see [15, Corollary 4.21, page 97) we obtain a continuous function $\tilde{\lambda}: M \rightarrow E$ such that $0<\tilde{\lambda}(p)<\beta(p) / 3 \leq \alpha(p) / 3$ for every $p \in M$.

Now we apply Theorem 4.2 with $\gamma=0, \lambda(p)=\min \{\tilde{\lambda}(p), \delta\}, \mathcal{C}(p)=x(p)+$ $2 \lambda(p) B$ and $\varepsilon(p)=4 L_{f}(p) \lambda(p)$, where $L_{f}(p)=\sup |f(p, u(p)+3 \lambda(p) B)| / \lambda(p)$. Note that $L_{f}$ is a Lipschitz constant of $f(p,$.$) on \mathcal{C}(p)$ (it follows from a basic lemma of convex analysis, see for instance [15], Lemma 5.23, page 742), so (22) is satisfied. By (iii) of Theorem 4.2 we obtain (b) and that $v(p)$ is an interior point of $\mathcal{C}(p)$, so putting $s(p)=-\Delta_{x}^{\prime}(p, v(p))$, from (v) of Theorem 4.2 we obtain (a). The assertion (c) follows from (a) and (b) if we take $M=E, u(p)=p$ and $f(p, x)=f(x)$.

Note that part (ii) (c) of the above corollary in finite-dimensional spaces is a better result than those obtained by applying Cellina's approximate selection theorem 7], Theorem 8.2.5, to the subdifferential of $f$. Cellina's theorem gives a continuous mapping $c: M \rightarrow E$ such that $c(y) \in \partial f(y+\delta B)+\delta B$.

Further we need the following lemma (see 14], Lemma 4.1) which shows the upper semi-continuity of the level-set mapping of a convex function.

Lemma 4.5. Let $X \subset E$ be a non-empty convex bounded subset, $f: E \rightarrow \mathbb{R}$ a convex function, $c \in \mathbb{R}, L(f, c):=\{x \in X: f(x) \leq c\}$, and let there exist $z \in X$ such that $f(z)<c$. Then for every $\varepsilon>0$ there exists $\delta>0$ such that $\operatorname{dist}_{L(f, c)}(x)<\varepsilon$ whenever $x \in X$ and $f(x)<c+\delta$.

By the next lemma we show that the assumption for existence of a continuous mapping $u$ satisfying (22) is fulfilled under some rather general conditions.

Lemma 4.6. Assume that the hypothesis $(\mathrm{H})$ is satisfied, $\mathcal{C}$ in addition is Hausdorff continuous with bounded images, the Slater condition (25) is satisfied and for every $p \in P$ the function $f(p,$.$) is bounded on the bounded subsets. Then there exists a$ continuous mapping $u: P \rightarrow C_{0}$ satisfying condition (22).

Proof. Claim: The multivalued mapping $L: P \rightarrow 2^{E}, L(p)=\left\{x \in C_{0}: g_{i}(p, x) \leq\right.$ $0, \forall i=1, \ldots, n\}$ is upper semi-continuous .

Let $p_{0} \in P$ and $\varepsilon>0$ be given. By Lemma 4.5, there exists $\delta>0$ such that

$$
L\left(g_{i}\left(p_{0}, .\right), \delta\right) \subset L\left(g_{i}\left(p_{0}, .\right), 0\right)+\varepsilon B, \quad \forall i=1, \ldots, n .
$$

By equi-lower semi-continuity, there exists an open set $\mathcal{O} \ni p_{0}$ such that

$$
g_{i}\left(p_{0}, x\right)<g_{i}(p, x)+\delta \quad \forall x \in C_{0}, p \in \mathcal{O}, i=1, \ldots, n .
$$

Take $p \in \mathcal{O}$ and $x \in L(p,$.$) . Then (32) and (33) imply x \in L\left(g_{i}\left(p_{0},.\right), \delta\right) \subset$ $L\left(g_{i}\left(p_{0},.\right), 0\right)+\varepsilon B$, which proves the Claim.

Note that $X_{g}(p)=L(p) \cap(\mathcal{C}(p)+\gamma(p) B)$ and, due to the Slater condition (25), int $X_{\gamma}(p) \neq \emptyset$ and $L$ is lower semi-continuous (therefore Hausdorff lower semicontinuous). Now Proposition 2.80 of [15, page 66, implies that $X_{\gamma}$ is Hausdorff continuous; therefore the multivalued mapping $X_{\gamma}^{\circ}$ with images $X_{\gamma}^{\circ}(p):=L^{\circ}(p) \cap$ $(\mathcal{C}(p)+\gamma(p) B)$ is Hausdorff continuous too, where $L^{\circ}(p)=\left\{x \in C_{0}: g_{i}(p, x)<\right.$ $0, i=1, \ldots, n\}$. 
We will prove that the function $\varphi(p)=\inf _{x \in X^{\circ}(p)} f(p, x)$ is lower semi-continuous. Take $p_{0} \in P, \varepsilon>0$, an open set $U \ni p_{0}$ such that $\mathcal{C}(p) \subset \mathcal{C}\left(p_{0}\right)+\varepsilon B$, $X_{\gamma}^{\circ}(p) \subset X_{\gamma}^{\circ}\left(p_{0}\right)+\varepsilon B$ and $f\left(p_{0}, x\right)-f(p, x)<\varepsilon, \forall x \in \mathcal{C}(U), \forall p \in U$. Let $K$ be a Lipschitz constant of $f\left(p_{0},.\right)$ on $\mathcal{C}\left(p_{0}\right)+\varepsilon B$ (such a $K$ exists by boundedness of $f\left(p_{0},.\right)$ on the bounded subsets and by a basic lemma of convex analysis, see for instance [15], Lemma 5.23, page 742). For any $p \in U$, take $x \in X_{\gamma}^{\circ}(p)$ and $y \in X_{\gamma}^{\circ}\left(p_{0}\right)$ such that $f(p, x)<\varphi(p)+\varepsilon$ and $\|x-y\|<\varepsilon$. Then

$$
\begin{aligned}
\varphi\left(p_{0}\right)-\varphi(p) & <f\left(p_{0}, y\right)-f(p, x)+\varepsilon \\
& <f\left(p_{0}, y\right)-f\left(p_{0}, x\right)+2 \varepsilon \\
& <K\|x-y\|+2 \varepsilon \\
& <\varepsilon K+2 \varepsilon,
\end{aligned}
$$

which proves the lower semi-continuity of the function $\varphi$.

Now we apply Lemma 2.1(b) with $F=X_{\gamma}^{\circ}, g=\varphi$ and finish the proof.

\section{REFERENCES}

[1] V.M. Alekseev, V.M. Tikhomirov and S.V. Fomin, Optimal Control, Contemporary Soviet Mathematics, R. Garmkrelidze (ed.) Consultants Bureau, New York and London (1987). MR 0924574 (89e:49002)

[2] J.-P. Aubin, I. Ekeland, Applied Nonlinear Analysis, A Wiley - Interscience Publ., Jonh Wiley and Sons, 1984. MR0749753 (87a:58002)

[3] J. Borwein and D. Preiss, A smooth variational principle with applications to subdifferentiability and differentiability of convex functions, Trans. Am. Math. Soc., 303 (1987), 517-527. MR0902782 (88k:49013)

[4] J. M. Borwein, Treiman, Jay S.; Zhu, Qiji J. Partially smooth variational principles and applications, Nonlinear Anal. 35 (1999), no. 8, Ser. B: Real World Applications, 1031-1059. MR $1707806(2000 \mathrm{j}: 49028)$

[5] J. M. Borwein, Zhu, Qiji J., Variational analysis in nonreflexive spaces and applications to control problems with $L^{1}$ perturbations, Nonlinear Anal. 28 (1997), no. 5, 889-915 MR.1422192(97k:49040)

[6] J. M. Borwein, Zhu, Qiji J., Viscosity solutions and viscosity subderivatives in smooth Banach spaces with applications to metric regularity, SIAM J. Control Optim. 34 (1996), no. 5, 15681591. MR 1404847 (97g:49037)

[7] J. M. Borwein and A. S. Lewis, Convex Analysis and Nonlinear Optimization. Theory and Examples, CMS Books in Mathematics, Springer, 2000. MR 1757448 (2001h:49001)

[8] F. H. Clarke, Optimization and Non-smooth Analysis, J.Wiley and Sons, 1983.

[9] R. Deville and G. Godefroy and V. Zizler, Un principle variationel utilisant des fonctions bosses, C.R. Acad. Sci. Paris, Serie I, 312 (1991), 281-286. MR1089715 (91j:49019)

[10] R. Deville and G. Godefroy and V. Zizler, A smooth variational principle with applications to Hamilton-Jacobi equations in infinite dimensions, J. Funct. Anal. 111, (1993), 197-212. MR:1200641 (94b:49010)

[11] R. Deville and G. Godefroy and V. Zizler, Smoothness and Renormings in Banach Spaces, Pitman Monographs No. 64, London: Longman, 1993. MR1211634 (94d:46012)

[12] I. Ekeland, On the variational principle, J. Math. Anal. Appl., 47, (1974), 324-353. MR0346619 (49:11344)

[13] I. Ekeland, Nonconvex minimization problems, Bull. Amer. Math. Soc., 1, (1979), 443-373. MR0526967 (80h:49007)

[14] P. Gr. Georgiev, The strong Ekeland variational principle, the strong drop theorem and applications, J. Math. Anal. Appl., 21 (1988), 1-21. MF0934428 (89c:46019)

[15] S. Hu, N. Papageorgiou, Handbook of Multivalued Analysis, Vol. I, Kluwer, Mathematics and Its Applications, 419, 1997. MR1485775 (98k:47001)

[16] E. Michael, Continuous selections I, Annals of Math., 63(1956), 361-382. MR0077107 $(17: 990 \mathrm{e})$ 
[17] R. R. Phelps, Convex functions, Monotone Operators and Differentiability, Lecture Notes in Mathematics, No. 1364.

[18] M. Sion, On general minimax theorems, Pacific J. Math. 8 (1958), 171-176. MR0097026 $(20: 3506)$

Department of Mathematics and Informatics, Sofia University "St. Kl. Ohridski", 5 James Bourchier Blvd., 1126 Sofia, Bulgaria

Current address: Department of Electrical and Computer Engineering and Computer Science, University of Cincinnati, ML 0030, Cincinnati, Ohio 45221-0030

E-mail address: pgeorgie@ececs.uc.edu 